\title{
Low-frequency expansion for probability amplitudes: An alternative approach to certain intramolecular dynamics problems
}

\author{
Walter Nadler and R. A. Marcus \\ Arthur Amos Noyes Laboratory of Chemical Physics, ${ }^{\text {a) }}$ California Institute of Technology, Pasadena, \\ California 91125
}

(Received 19 December 1986; accepted 3 March 1987)

\begin{abstract}
We present an algorithm to determine the averaged time evolution of the probability amplitude for a nonstationary state in a quantum mechanical system. The algorithm is based on a lowfrequency expansion of the probability amplitude and is related to the generalized moment expansion method which has been applied successfully to the description of dynamic correlation functions in stochastic systems. It is shown that the proposed algorithm gives excellent results for the description of quantum beats in the time evolution of the occupation probability for a nonstationary state in model systems. The relation of the algorithm to other theoretical approaches and the relevance for the description of intramolecular energy transfer processes is discussed.
\end{abstract}

\section{INTRODUCTION}

Despite the already considerable complexity of a quantum Hamiltonian describing an isolated polyatomic molecule, the experimentally observed behavior of the time evolution of an initially prepared vibrational or vibronic state in such a system is often very simple. In experiments pioneered by Zewail and co-workers ${ }^{1,2}$ either an oscillatory time behavior of the occupation probability, i.e., "quantum beats," 1 or an approximately exponential decay, ${ }^{2}$ or both, ${ }^{1,2}$ were encountered. The former behavior corresponds to an oscillatory flow of energy between the prepared state and the rest of the molecule, and the latter to a quasidissipative redistribution of energy within the molecule. The relative simplicity of the physical situation - an isolated polyatomic moleculeand the relative simplicity of the observed behavior, which are in contrast to the complexity of dynamics when many states are involved, pose a particular challenge for the theoretical description of the quantum dynamics of a nonstationary state in such systems. ${ }^{3}$

In the present paper we concentrate on the situation where an oscillatory flow of energy between an observed state and the remaining states of the molecule occurs and present a simple method to describe these quantum beats quantitatively starting from a given Hamiltonian. The physical situation and the method are described in Sec. II. In Sec. III we consider several model systems and compare the exact solution with the present approximation method. In Sec. IV we discuss the quality of the method and compare it with the other approaches. In the Appendices several equivalent formulations of the method are given: continued fracton expansion, an equivalent reduced Hamiltonian description, a related simpler eigenvalue problem, and the method of inner projections.

\section{THEORY}

It is a well-known quantum mechanical text book problem to determine the evolution of a nonstationary state. Nev-

\footnotetext{
a) Contribution No. 7526.
}

ertheless, it is useful to repeat it here in order to clarify the idea of our method.

A quantum mechanical system can be prepared, for example by a pulse of light, in a state $|\psi(0)\rangle$ which is not an eigenstate of the Hamiltonian $\mathbf{H}$. The time evolution of $|\psi(t)\rangle$ is determined by the Schrödinger equation

$$
i \frac{d}{d t}|\psi(t)\rangle=\mathbf{H}|\psi(t)\rangle
$$

and can be described through a spectral expansion

$$
|\psi(t)\rangle=\sum_{j}\left\langle\varphi_{j} \mid \psi(0)\right\rangle e^{-i E_{j} t}\left|\varphi_{j}\right\rangle,
$$

with the $\left|\varphi_{j}\right\rangle$ being eigenstates of $\mathbf{H}$ with energy $E_{j}$. From Eq. (2.2) all temporal properties of the quantum system can be derived. In pump-probe experiments ${ }^{4}$ one is frequently interested in the projection of $|\psi(t)\rangle$ on some other wave function, e.g., on a dipole operator which, in turn, is operating on some particular vibronic state. In the present paper, to illustrate and test the method, we shall consider the probability amplitude for the system being observed in the initial state $|\psi(0)\rangle$,

$$
b(t)=\langle\psi(0) \mid \psi(t)\rangle=\sum_{j} b_{j} e^{-i E_{j} t}
$$

This projection is also of experimental interest. ${ }^{1,2}$ The expansion coefficients $b_{j}$ are the square modulus of the overlap between the initial state and the eigenstate $\left|\varphi_{j}\right\rangle$, i.e.,

$$
b_{j}=\left|\left\langle\varphi_{j} \mid \psi(0)\right\rangle\right|^{2} .
$$

In a subsequent paper more general projections than that in Eq. (2.3a) will be treated. We note that the quantity actually observed is not the probability amplitude (2.3a) but the occupation probability itself,

$$
\begin{aligned}
& p(t)=|b(t)|^{2}=\sum_{j} b_{j}^{2}+\sum_{\substack{j, k \\
j \neq k}} b_{j} b_{k} \cos \left(\omega_{j k} t\right), \\
& \text { with } \omega_{j k}=\left|E_{j}-E_{k}\right| .
\end{aligned}
$$

The spectral expansion (2.2) of the wave function and, consequently, the occupation probability (2.4) of the non- 
stationary state can be determined numerically from a diagonalization of the Hamiltonian $H$. However, for the case of an, actual polyatomic molecule this poses some practical problems. Even for an approximate matrix Hamiltonian with the dimension of the Hilbert space truncated to a finite but large number of functions, the numerical task of the diagonalization is quite considerable. A number of methods have been developed to deal with such a problem. From the numerical side come methods for the determination of the eigenvalues of large (possibly sparse) matrices on high speed parallel computers by employing the Lanczos algorithm, ${ }^{5}$ which is also employed implicitly in the recursive residue generation method. ${ }^{6}$ Other approaches represent simplifications of the problem by reducing the function space to include only such functions that appear relevant, a property to be determined by, e.g., artificial intelligence methods. ${ }^{7} \mathrm{~A}$ further reduction can be achieved by concentrating on a still smaller subset of (possibly resonant) states where the self-energy and the interactions of these states are renormalized by the neglected states, giving rise to effective coupled equations based on an effective Hamiltonian. ${ }^{8}$

An approach to determine the spectral expansion (2.2) as correctly as possible seems to provide more information than is actually needed. What can be observed reliably in an experiment is some average behavior, e.g., the low-frequency quantum beats inherent in Eq. (2.4). An approximation that describes mainly the low-frequency behavior of $p(t)$ is desirable, therefore, and it should, in appropriate cases, be achievable in a way computationally easier than through a diagonalization of the full Hamiltonian $\mathbf{H}$.

The low-frequency behavior of $p(t)$ in Eq. (2.4) is determined by those states $\left|\varphi_{j}\right\rangle$ of $\mathrm{H}$ whose energies $E_{j}$ lie close to each other. In addition, in order to give rise to noticeable low-frequency quantum beats, those states should have an appreciable overlap with the initial state $|\psi(0)\rangle$ in the present problem, so that they can give a noticeable contribution to $p(t)$. A good first guess is that such states are energetically near to the energy of the initial state, given by

$$
\langle\mathbf{H}\rangle=\langle\psi(0)|\mathbf{H}| \psi(0)\rangle=\sum_{j} b_{j} E_{j},
$$

since the larger terms in the distribution $b_{j}$ determine $\langle\mathbf{H}\rangle$. Hence, our working hypothesis is that the low-frequency behavior of $p(t)$ is given mainly by the spectral properties of $b(t)$ around $\langle\mathbf{H}\rangle$. We desire, therefore, an approximation to $b(t)$ that reproduces the exact spectral properties of $b(t)$ around $\langle\mathbf{H}\rangle$.

To accomplish such an approximation we first separate a time-dependent phase factor with frequency $\langle\mathbf{H}\rangle$ from $b(t)$ and obtain a transformed probability amplitude

$$
C(t)=e^{i\langle\mathbf{H}\rangle t} b(t) .
$$

This transformation amounts to a shift of the zero of the spectrum of $b(t)$ to $\langle\mathbf{H}\rangle$. For the actual observable, $p(t)$, this shift is of no relevance and the $p(t)$ in Eq. (2.4) is equivalently given as the square modulus of the $C(t)$ in Eq. (2.6). Employing the shifted Hamiltonian

$$
\delta \mathbf{H}=\mathbf{H}-\langle\mathbf{H}\rangle,
$$

the probability amplitude $C(t)$ can be written formally as the matrix element of an exponential operator

$$
C(t)=\left\langle\psi(0)\left|e^{-i \delta \mathbf{H} t}\right| \psi(0)\right\rangle \text {. }
$$

Upon using Laplace transformation in the form

$$
\bar{C}(\omega)=\lim _{\eta \rightarrow 0^{+}} i \int_{0}^{\infty} d t e^{-(i \omega+\eta) t} C(t),
$$

where $\boldsymbol{\eta}>0$ is introduced to ensure convergence, we can write $\bar{C}(\omega)$ in a form comparable to Eq. $(2.8)$, as a matrix element of the resolvent operator $[\omega+\delta \mathbf{H}]^{-1}$ :

$$
\bar{C}(\omega)=\left\langle\psi(0)\left|[\omega+\delta \mathbf{H}]^{-1}\right| \psi(0)\right\rangle .
$$

A formal expansion for $\bar{C}(\omega)$ around $\omega=0$ yields

$$
\bar{C}(\omega) \sim \sum_{n=0}(-\omega)^{n} \mu_{-(n+1)},
$$

with the expansion coefficients $\mu_{-n}$ given as matrix elements of the inverse shifted Hamiltonian $\delta \mathbf{H}$,

$$
\mu_{-n}=\left\langle\psi(0)\left|\delta \mathbf{H}^{-n}\right| \psi(0)\right\rangle .
$$

A method for calculating these low-frequency moments $\mu_{-n}$ for an actual shifted Hamiltonian $\delta \mathbf{H}$ is given below [Eqs. (2.20) to (2.22)]. The low-frequency moments are employed as follows for the construction of an $N$ th-order approximation $\bar{c}_{N}(\omega)$ to $\bar{C}(\omega)$ that reproduces the low-frequency properties of the exact function $\bar{C}(\omega)$ :

For the functional form of the approximation we choose $\bar{c}_{N}(\omega)$ to be meromorphic:

$$
\bar{c}_{N}(\omega)=\sum_{j=1}^{N} \frac{c_{j}}{\omega+\epsilon_{j}},
$$

which is equivalent to an [ $(N-1) / N]$-Padé approximation ${ }^{9}$ to $\bar{C}(\omega)$. In the time domain this form corresponds to a finite number of exponentials,

$$
c_{N}(t)=\sum_{j=1}^{N} c_{j} e^{-i \epsilon_{j} t},
$$

a truncated version of Eq. (2.3) signifying that we are only interested in a few frequencies. The approximation $\bar{c}_{N}(\omega)$ has to reproduce the low-frequency moments $\mu_{-1}$ to $\mu_{-2 N}$ to have the correct low-frequency properties of the exact function $\bar{C}(\omega)$. This property requires that the parameters $c_{j}$ and $\epsilon_{j}$ of Eq. (2.13) satisfy the nonlinear relations

$$
\sum_{j=1}^{N} c_{j} \epsilon_{j}^{-n}=\mu_{-n}, \quad n=1, \ldots, 2 N
$$

Parenthetically we note that Eq. (2.13a) for $\bar{c}_{N}(\omega)$ can be written also as a truncated continued fraction (as in Appendix A), and so an alternative method for the determination of the parameters $c_{j}$ and $\epsilon_{j}$ could have been based on that approach.

From the form of the approximation (2.13b) one can well imagine that there exists some "equivalent Hamiltonian" form whose eigenvalues are the $\epsilon_{j}$ 's. This equivalent formulation is established in Appendix B, where an equivalent reduced symmetric Hamiltonian is derived with real eigenvalues $\epsilon_{j}$ and eigenvectors leading to real positive val- 
ues for the $c_{j}$ (Appendices B 1 to B 3). In Appendix B 4, several properties of the equivalent "reduced" initial state for this reduced Hamiltonian are discussed.

For $N=1$ and $N=2$, the algebraic solution of Eqs. (2.14) is straightforward and the results are given by

$$
\begin{aligned}
& \epsilon_{1}=\mu_{-1} / \mu_{-2}, \\
& c_{1}=\mu_{-1}^{2} / \mu_{-2}
\end{aligned}
$$

for $N=1$, and by

$$
\begin{aligned}
\epsilon_{1,2}= & \frac{1}{2} \frac{1}{\left(\mu_{-2} \mu_{-4}-\mu_{-3}^{2}\right)}\left[\left(\mu_{-1} \mu_{-4}-\mu_{-2} \mu_{-3}\right)\right. \\
& \left. \pm \sqrt{\left(\mu_{-1} \mu_{-4}-\mu_{-2} \mu_{-3}\right)^{2}-4\left(\mu_{-1} \mu_{-3}-\mu_{-2}^{2}\right)\left(\mu_{-2} \mu_{-4}-\mu_{-3}^{2}\right)}\right],
\end{aligned}
$$

$$
c_{1,2}= \pm \frac{\left(\mu_{-1}-\epsilon_{2,1}\right) \epsilon_{1,2}^{2}}{\epsilon_{1}-\epsilon_{2}}
$$

for $N=2$. These two approximations to $C(t), c_{1}(t)$ and $c_{2}(t)$, are the simplest ones and we shall discuss their properties and the consequences for the approximate observable $p_{N}(t)$ shortly.

For the first-order approximation, $N=1$, Eq. $(2.13 \mathrm{~b})$ is a single complex phase factor and, hence, it does not lead to any dynamic information about the occupation probability $p(t)$. However, it gives already a first estimate for the timeaveraged probability $\bar{p}$ of finding the system in the initial state,

$$
\begin{aligned}
p_{1}(t) & =p_{0} \\
& \approx \bar{p}=\lim _{T \rightarrow \infty} \frac{1}{T} \int_{0}^{T} d t p(t)
\end{aligned}
$$

with $p_{0}=c_{1}^{2}$. To obtain some dynamic information one has to go to the second-order approximation, $N=2$, which is the lowest order of an approximation that can describe quantum beats. The corresponding approximation to the occupation probability is

$$
p_{2}(t)=p_{0}+p_{1} \cos \left(\omega_{1} t\right),
$$

with $\omega_{1}=\left|\epsilon_{1}-\epsilon_{2}\right|$ being the frequency of the quantum beat, $p_{0}=c_{1}^{2}+c_{2}^{2}$, and $p_{1}=2 c_{1} c_{2}$.

For $N>2$ an algebraic solution of the nonlinear equations (2.14) becomes complicated. We then employ an equivalent eigenvalue problem, related to the reduced Hamiltonian form of Appendix B and described in Appendix C, for the actual numerical determination of the $\epsilon_{j}$ and $c_{j}$. Higher-order approximations to $C(t)$ lead to forms for $p_{N}(t)$ more complicated than Eqs. (2.17) and (2.18), and for those we will use the notation, corresponding to Eq. (2.18):

$$
p_{N}(t)=p_{0}+\sum_{v=1}^{N(N-1) / 2} p_{v} \cos \left(\omega_{v} t\right),
$$

where the $\omega_{v}$ are assumed to be ordered, i.e., $\omega_{1} \leqslant \omega_{2} \leqslant \cdots$.

With increasing $N$ the approximation $\bar{c}_{N}(\omega)$ converges to the exact function $\bar{C}(\omega)$, as discussed in Appendix $D$, and, hence, $p_{N}(t)$ will ultimately converge to the exact $p(t)$, too.

We note at this point that the approximation method presented above is very similar to the generalized moment expansion method. ${ }^{10}$ This method has been applied successfully for the description of various correlation functions in stochastic systems. ${ }^{10,11}$ In the above treatment, we have uti- lized, in effect, the equivalence between a real-time evolution of a stochastic equation and the imaginary-time evolution of a Schrödinger equation. ${ }^{12}$

In the remaining part of this section we discuss the actual determination of the generalized moments $\mu_{-n}$. Although, as we shall see, the method employed is very simple in principle, it should be noted that the main numerical task encountered in the application of the present method is a numerically correct determination of the low-frequency moments.

We first introduce auxiliary functions $\left|\mu_{-n}\right\rangle$ through

$$
\left|\mu_{-n}\right\rangle=\delta \mathbf{H}^{-n}|\psi(0)\rangle \text {. }
$$

These auxiliary functions can be calculated successively for increasingly higher $n$ by solving the equations

$$
\delta \mathbf{H}\left|\mu_{-n}\right\rangle=\left|\mu_{-(n-1)}\right\rangle .
$$

for $\left|\mu_{-n}\right\rangle$ with $\left|\mu_{0}\right\rangle=|\psi(0)\rangle$ as starting rhs function.

For the Hamiltonian of a polyatomic molecule Eq. (2.21) is a high-dimensional elliptic differential equation. Either by a discretization of this Hamiltonian ${ }^{10(c), 13}$ or, and this seems more appropriate for actual applications, by a suitable expansion in orthogonal functions, one can usually get a very good description of the exact Hamiltonian in terms of a high-order matrix Hamiltonian. For a matrix Hamiltonian, Eq. (2.21) constitutes a set of linear equations the numerical solution of which is straightforward. ${ }^{13}$ We have solved Eqs. (2.21) using standard linear equation solvers available on every computer. In case the linear equations are determined by large sparse matrices, appropriate numerical routines also available on modern large memory high speed computers can be used [see related references in Ref. 10 (c) ]. We note that probably also the powerful Lanczos method $^{5}$ of tridiagonalizing a matrix may be used to solve the linear equations ( 2.21 ).

The actual moments $\mu_{-n}$ are then calculated from the such determined auxiliary functions $\left|\mu_{-n}\right\rangle$ simply through their scalar products, i.e.,

$$
\begin{aligned}
& \mu_{-(2 n-1)}=\left\langle\mu_{-(n-1)} \mid \mu_{-n}\right\rangle, \\
& \mu_{-2 n}=\left\langle\mu_{-n} \mid \mu_{-n}\right\rangle .
\end{aligned}
$$

In the examples to be considered in the next section our starting point is always already a matrix form of the Hamiltonian $\mathbf{H}$.

\section{APPLICATION TO MODEL SYSTEMS}

The first model we shall discuss is a 13 -state system with a four-state resonant subspace which includes the initial 
nonstationary state. This model was considered in Ref. 8(a), Sec. II B, and we refer the interested reader to Table I of this reference for the exact Hamiltonian. We also consider the related model discussed there, where the four-state resonant subspace is replaced by a three-state resonant subspace (which includes the initial state) plus one off-resonant state, the Hamiltonian having been given in Table II of the above reference. The time dependence of the probability of the initial state $p(t)$ for these systems is shown in Fig. 1, calculated by the present approximation, with $N=2$, and by the exact solution of the system via a spectral expansion. The numerical results for the parameters of the approximation [the $p_{0}$, $p_{1}$, and $\omega_{1}$ in Eqs. (2.17) and (2.18)] are given in Table $I$. One sees from Fig. 1 that in both cases a second-order approximation is already sufficient to reproduce the low-frequency quantum beats in each model. As Table I also demonstrates, the third-order approximation gives only negligible corrections to the amplitude and the frequency of these oscillations.

In Fig. 2 it is shown how higher-order approximations

model 1a

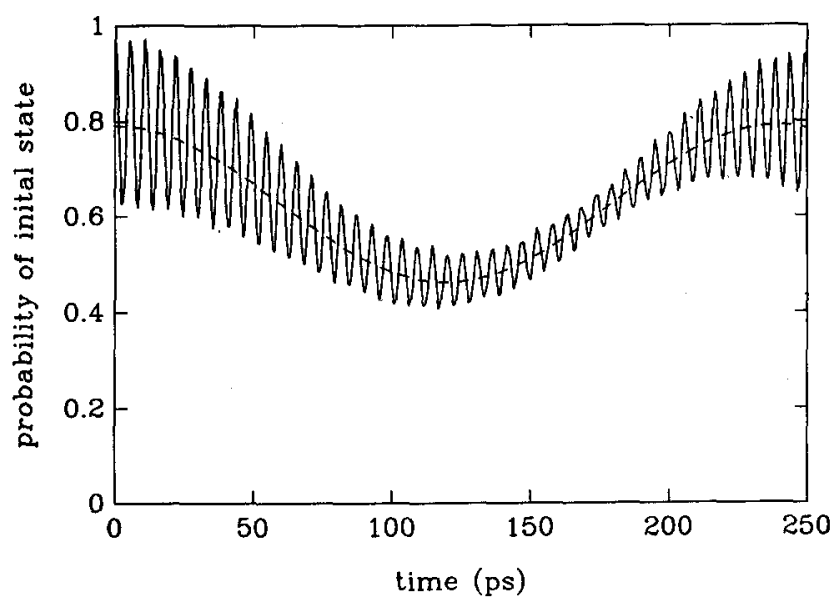

model $1 \mathrm{~b}$

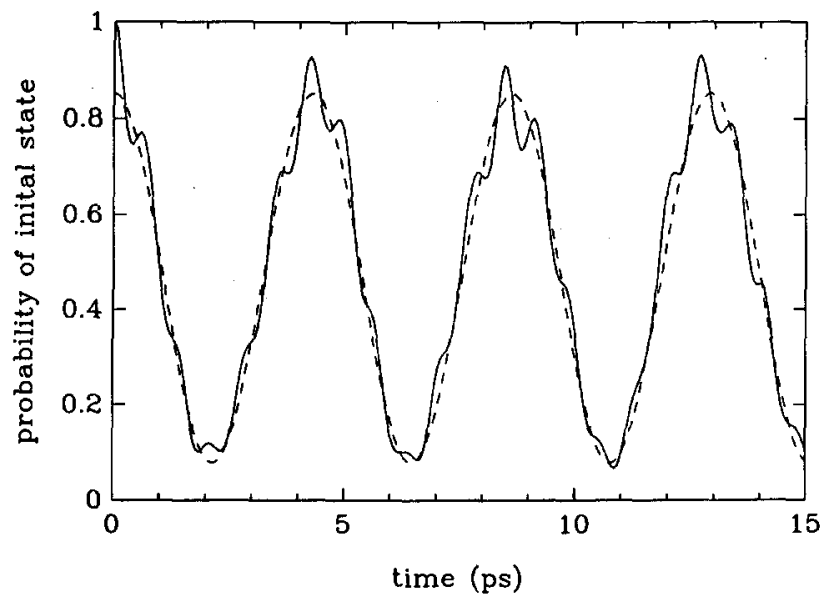

FIG. 1. Probability $p(t)$ for the initial state $|\psi(0)\rangle$ vs $t$ for the 13-state model system given in Ref. 8(a), Sec. II B: (a) Table I of that reference, (b) Table II of that reference. (-) is the exact solution, $(--)$ is the secondorder approximation, i.e., $N=2$.
TABLE I. Parameters for the lowest mode in the low-frequency approximation for $p(t)$ of model 1 .

\begin{tabular}{lcccc}
\hline & & $p_{0}$ & $p_{1}$ & $\omega_{1}\left(\mathrm{~cm}^{-1}\right)$ \\
\hline (a) & & & & \\
& $N=1$ & 0.038 & $\ldots$ & $\ldots$ \\
& $N=2$ & 0.6268 & 0.1644 & 0.1391 \\
& $N=3$ & 0.6261 & 0.1644 & 0.1391 \\
(b) & & & & \\
& $N=1$ & $1.8 \times 10^{-6}$ & $\ldots$ & $\ldots$ \\
& $N=2$ & 0.466 & 0.387 & 7.732 \\
& $N=3$ & 0.477 & 0.387 & 7.755 \\
\hline
\end{tabular}

describe the more complicated features in the time evolution of $p(t)$ in the above models. It can be seen that the high frequency oscillations superimposed on the dominant quantum beats are described reasonably well by a fourth-order approximation, though some details are still missing. That one has to go to a higher- (here fourth-) order approximation to get results for these higher-frequency beats can simply be attributed to the fact that the higher-frequency oscilla-
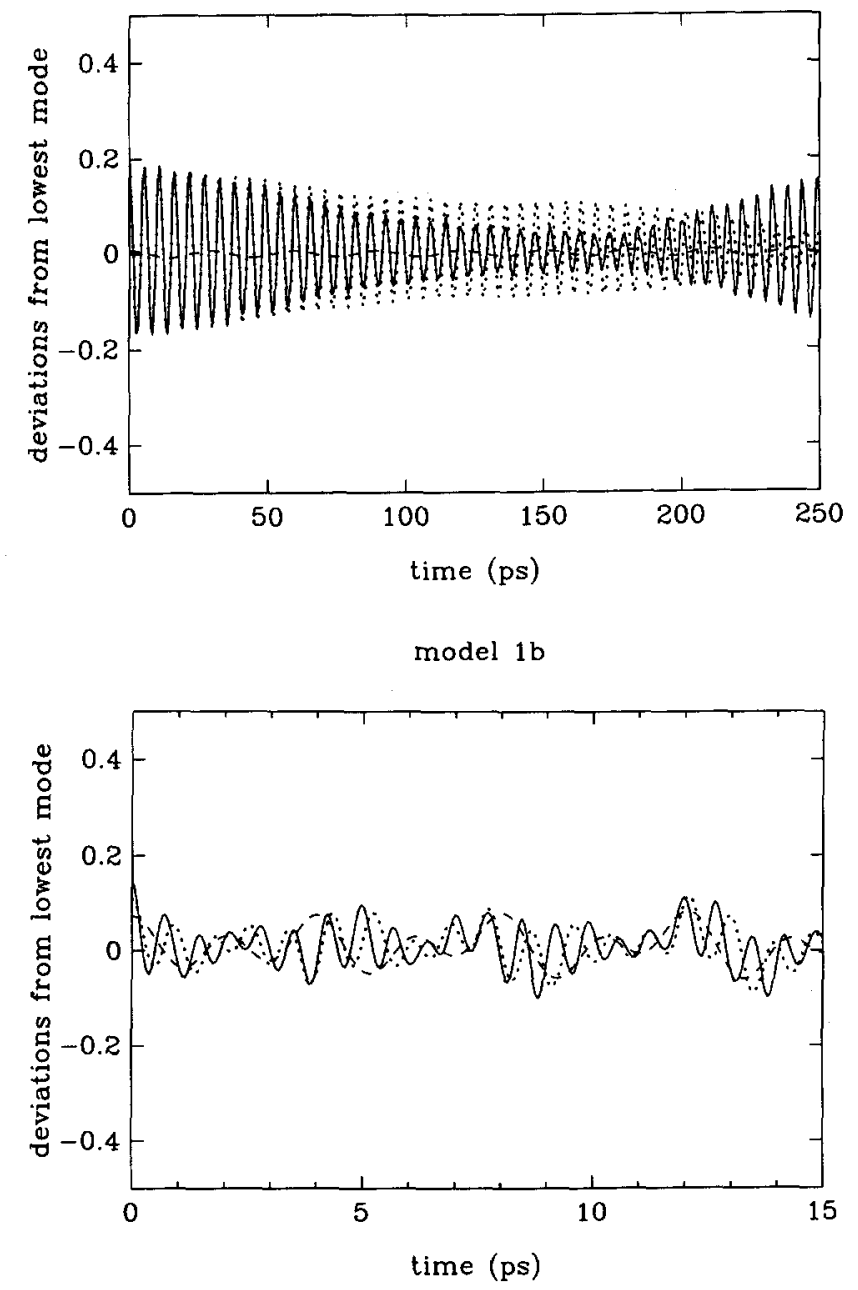

FIG. 2. Deviation of the probability $p(t)$ from the second-order approximation for the models shown in Fig. 1 (a) and 1(b). (-) is the exact solution, $(--)$ is the third-order approximation, i.e., $N=3,(\cdots)$ is the fourth-order approximation, i.e., $N=4$. 
tions are determined by modes of the exact Hamiltonian that are relatively far from the states that contribute to the lowest dominant quantum beat mode. Hence, a low-frequency expansion to an appropriate order is necessary to take into account these contributions. This feature may also explain the observation that the fourth-order approximation in model $1 \mathrm{~b}$ is somewhat better than in model 1a: the frequency of the superimposed oscillations in model $1 \mathrm{~b}$ is relatively lower than in model la.

A common property of the above models, seen from the values for $p_{0}$ in Table $\mathrm{I}$, is that the first-order approximations, $N=1$, give erroneous values for the long-time averaged probability $\bar{p}$ of the initial state. This property may well be related to the fact that both models feature subspaces with states resonant or almost resonant with the initial state. Since there are two classes of bath states that influence the time evolution, the resonant and the nonresonant states, one has to take into account more complicated features of the internal dynamics that cannot be accounted for in a firstorder approximation. However, as we have seen in Fig. 1, a second-order approximation is already sufficient to reproduce both the dominant low-frequency quantum beats and the time-averaged behavior.

In our last example we shall consider a model system with no resonant subspace of states. We choose a 21-state random Hamiltonian with diagonal elements $H_{11}=0$, $10.0 \leqslant\left|H_{i i}\right| \leqslant 100.0 \mathrm{~cm}^{-1}(i \neq 1)$, and off-diagonal elements $0.0 \leqslant\left|H_{i j}\right| \leqslant 2.0 \mathrm{~cm}^{-1}(i \neq j)$. In Fig. 3 the time dependence of the probability of the initial state, $p(t)$, for this model is plotted, calculated by our approximation, with $N=2$, and by the exact solution of the system via a spectral expansion. The numerical results for the parameters of the approximation $\left(p_{0}, p_{1}\right.$, and $\left.\omega_{1}\right)$ are given in Table II. In this case, even the first-order approximation is seen to reproduce the longtime average $p_{0}$ of the probability of the initial state, correctly. The result for the low-frequency quantum beats in the second-order approximation is also excellent. These results support the above considerations concerning the influence of resonant subspaces on the quality of the first-order approximation.

It is seen that in the last example, unlike the systems in Fig. 1 , the probability oscillates between values $(\approx 0.92$, $\approx 0.99$ ) that are close to unity. Near-resonant states or a strong coupling to off-resonant states is needed to allow this probability to become small. Both properties are absent, by construction, in the model system that led to Fig. 3.

In closing this section we note that an application of the present algorithm to very small systems proves to be ineffective. For example, for a four-state system one may well have to go to a third-order approximation to obtain reasonable results for the dominant low-frequency quantum beats. In contrast, in systems with a large number of contributing states the particular contributions from individual states are more or less averaged out and a second-order approximation is already sufficient to reproduce the dominant quantum beats.

\section{DISCUSSION}

The above examples illustrate that quantum beats in the time evolution of the occupation probability for an initially model $2 /$ random hamiltonian

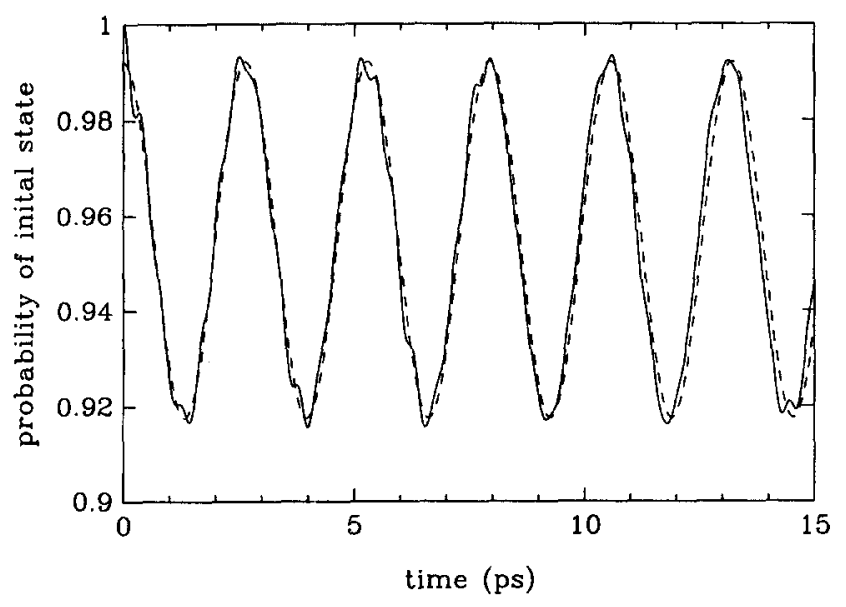

FIG. 3. Probability $p(t)$ for the initial state $|\psi(0)\rangle$ vs $t$ for the 21-state random model system described in the text. $(-)$ is the exact solution, (- ) is the second-order approximation, i.e., $N=2$.

prepared nonstationary state can be described by the present method with an approximation of very low order. Thereby, the method provides a useful tool to determine in a simple way the frequency and amplitude of such quantum beats from a known molecular Hamiltonian or its matrix representation. By proceeding to higher-order approximations one can also tailor the accuracy of the approximation to the accuracy of the data available, e.g., when the superimposed high frequency oscillations are distinguishable experimentally.

We consider next the computational expense of the present method as compared with that of other approaches. When the full Hamiltonian is expanded in suitably chosen orthogonal functions to construct a matrix Hamiltonian, there are mainly two other methods: (i) the diagonalization of the full Hamiltonian matrix and (ii) the derivation of an effective Hamiltonian for a reduced subspace. An interesting feature of the present method is that the computation time grows only linearly with the order $N$ of the approximation, the time-limiting step being the determination of the lowfrequency moments. To determine the properties of the lowest frequency quantum beats in the time evolution of the occupation probability a second-order approximation (or a third-order approximation for a check of the result) was shown to be sufficient. For these approximations the determination of the auxiliary functions $\left|\mu_{-1}\right\rangle$ to $\left|\mu_{-2}\right\rangle$ (or to $\left.\left|\mu_{-3}\right\rangle\right)$ is necessary, which amounts to the solution of two (or three) sets of linear equations. The computation time is,

TABLE II. Parameters for the lowest mode in the low-frequency approximation for $p(t)$ of model 2 .

\begin{tabular}{cccc}
\hline & $p_{0}$ & $p_{1}$ & $\omega_{1}\left(\mathrm{~cm}^{-1}\right)$ \\
\hline$N=1$ & 0.9534 & $\ldots$ & $\ldots$ \\
$N=2$ & 0.9549 & 0.0373 & 12.61 \\
$N=3$ & 0.9549 & 0.0372 & 12.74 \\
\hline
\end{tabular}


therefore, much smaller than for the determination of all eigenvalues, even if one takes into account that the (iterative) solution of linear equations is usually slower than the iterative determination of a single eigenvalue. Details, however, depend on the actual numerical routines employed. The computation time would be also smaller than for the determination of effective Hamiltonians when for the latter task inversions of a large portion of the full Hamiltonian matrix are necessary. We note that in suitable situations an iterative determination of these inverse submatrices is possible. $^{8(b), 8(c)}$

One could argue that a specific evaluation of eigenvalues in a particular part of the spectrum of $\delta \mathrm{H}$, say around zero, is equivalent to our approach. However, we note that also in such an approach the eigenvalues and eigenvectors are determined irrespective of how relevant they are for the description of the particular observable, the probability amplitude $C(t)$ in the present case. The computational strength of the present method lies in the fact that it samples the specific spectral properties already of $C(t)$, and not of $\delta \mathbf{H}$. Thereby, a satisfactory description of $C(t)$ can be reached already at a low-order approximation, whereas in the above eigenvalue approach a large number of eigenvalues may have to be sampled to find the relevant ones.

The main advantage of the present method lies in the simple determination of the dominant low-frequency quantum beat mode. In addition, the present method treats all Hamiltonians on an equal footing, independent of their respective structure, and is therefore model independent. It is, for example, unnecessary to inspect the Hamiltonian to determine a suitable subspace for which, in turn, an effective Hamiltonian can be determined. ${ }^{8}$ In a sense, the present method chooses itself a suitable reduced space with the dimension $N$ given by the order of the approximation (cf. Appendix $B$ ), i.e., given by the desired accuracy of the approximation.

We note that the present approach does not in itself elucidate the pathways of intramolecular energy flow. This is an area where the effective Hamiltonian approach in Ref. 8 provides information of a complementary nature. A given Hamiltonian may be analyzed first by the present method to obtain information about the dynamics of the initial nonstationary state. This information on whether or not significant quantum beats exist, and the information present in the parameters and structure of the reduced space spanned by a set of vectors $|k\rangle$, described in Appendix $\mathbf{D}$, can then also be utilized for an inspection the Hamiltonian matrix elements to determine the principal states contributing to these beats. Employing this result, an effective Hamiltonian for the relevant subspace can be formulated by the method of Ref. 8., so adding to the physical insight into the origin of the beats for the particular system. For example, after obtaining the results in Fig. 3 subsequent (unpublished) calculations by Voth showed that the effective Hamiltonian of Ref. 8 could be used to treat the behavior of model 2, using a particular two-state subspace and treating the remaining states as bath states.

We are currently working on an extension of the present method to describe more complicated observables than the probability amplitude in Eq. (2.3). By considering time-dependent probability amplitudes for the transition between different states as observables, this extension could also provide a further answer to the above question about intramolecular pathways of energy transfer.

The present method does not attempt to describe the quasidissipative decay encountered also in experiments. We are exploring the possible extension of the method also for this purpose, taking into account the specific experimental situation.

\section{ACKNOWLEDGMENTS}

W. N. likes to thank G. A. Voth and S. J. Klippenstein for introducing him to the problem and for interesting discussions. We also thank G. A. Voth for a critical reading of the manuscript. This work has been supported by a grant from the National Science Foundation.

\section{APPENDIX A: CONTINUED FRACTION EXPANSION}

For a function $I(\omega)$ with a known formal expansion for large $\omega$,

$$
I(\omega) \sim \frac{1}{\omega} \sum_{n=0}^{\infty}\left(-\frac{1}{\omega}\right)^{n} \mu_{n}
$$

a corresponding continued fraction

$$
C^{(h)}(\omega)=\frac{\alpha_{1}}{\omega+\frac{\alpha_{2}}{1+\frac{\alpha_{3}}{\omega+\frac{\alpha_{4}}{1+\cdots}}}}
$$

can be constructed ${ }^{14}$ with $C^{(h)}(\omega)$ fulfilling the requirement that its expansion coefficients in powers of $1 / \omega$ are equal to the moments $\mu_{n}$ in Eq. (A1). The coefficients $\alpha_{v}$ are given through the moments $\mu_{n}$ by ${ }^{14}$

$$
\begin{aligned}
& \alpha_{1}=\mu_{0}, \\
& \alpha_{2}=\mu_{1} / \mu_{0}, \\
& \alpha_{3}=\left(\mu_{0} \mu_{2}-\mu_{1}^{2}\right) / \mu_{0} \mu_{1}, \\
& \alpha_{4}=\left(\mu_{1} \mu_{3}-\mu_{2}^{2}\right) \mu_{0} /\left(\mu_{0} \mu_{2}-\mu_{1}^{2}\right) \mu_{1}, \\
& \vdots
\end{aligned}
$$

which can be verified by comparison of the respective expansion coefficients.

An $N$ th-order approximant to $I(\omega)$ is a truncated continued fraction $C_{N}^{(h)}(\omega)$ where the coefficient $\alpha_{N+1}$ in Eq. (A2) has been set to zero. Of particular interest in our case are even approximants $C_{2 N}^{(h)}(\omega)$ which can be cast into the form $^{14}$

$$
C_{2 N}^{(h)}(\omega) \quad \frac{\alpha_{1}}{\omega+\alpha_{2}-\frac{\alpha_{2} \alpha_{3}}{\omega+\left(\alpha_{3}+\alpha_{4}\right)-\frac{\alpha_{4} \alpha_{5}}{\omega+\left(\alpha_{5}+\alpha_{6}\right)-\cdots}}}
$$


and constitute $[(N-1) / N]$-Padé approximants to $I(\omega) .{ }^{15}$ Padé approximants of the continued fraction form (A2) and determined according to the scheme provided by Eqs. (A1) to (A4) have had a widespread application in various physical situations, e.g., in the well-known Mori-Zwanzig projection operator method ${ }^{16}$ for the description of dynamical correlation functions.

By employing the above results it is now easy to construct a similar continued fraction for a function with a known formal expansion for small arguments, as is the case in Eq. (2.11). We note that the replacement $I(\omega) \rightarrow(1 /$ $\omega) I(1 / \omega)$ and $\mu_{n} \rightarrow \mu_{-(n+1)}$ in Eq. (A1) gives the formal expansion

$$
\frac{1}{\omega} I\left(\frac{1}{\omega}\right) \sim \sum_{n=0}^{\infty}(-\omega)^{n} \mu_{-(n+1)},
$$

a form similar to Eq. (2.11). Hence, a continued fraction $C^{(l)}(\omega)$, with its expansion coefficients in powers of $\omega$ equal to the moments $\mu_{-(n+1)}$ in Eq. (A5), is given by

$$
\begin{aligned}
C^{(l)}(\omega) & =\frac{1}{\omega} C^{(h)}\left(\frac{1}{\omega}\right) \\
& =\frac{\alpha_{1}}{1+\frac{\alpha_{2} \omega}{1+\frac{\alpha_{3} \omega}{1+\frac{\alpha_{4} \omega}{1+\cdots}}}}
\end{aligned}
$$

The coefficients $\alpha_{v}$ in Eq. (A6) are calculated from Eqs. (A3) simply by the above replacement $\mu_{n} \rightarrow \mu_{-(n+1)}$, resulting in

$$
\begin{array}{ll}
\alpha_{1}=\mu_{-1}, & \text { (A7a) } \\
\alpha_{2}=\mu_{-2} / \mu_{-1}, & \text { (A7b) } \\
\alpha_{3}=\left(\mu_{-1} \mu_{-3}-\mu_{-2}^{2}\right) / \mu_{-1} \mu_{-2}, & \text { (A7c) } \\
\alpha_{4}=\left(\mu_{-2} \mu_{-4}-\mu_{-3}^{2}\right) \mu_{-1} /\left(\mu_{-1} \mu_{-3}-\mu_{-2}^{2}\right) \mu_{-2},
\end{array}
$$

An even approximant $C_{2 N}^{(l)}(\omega)$ assumes the form

$$
C_{2 N}^{(l)}(\omega)=\frac{\alpha_{1}}{1+\alpha_{2} \omega-\frac{\alpha_{2} \alpha_{3} \omega}{1+\left(\alpha_{3}+\alpha_{4}\right) \omega-\frac{\alpha_{4} \alpha_{5} \omega}{1+\left(\alpha_{5}+\alpha_{6}\right) \omega-\cdots}}}
$$

$C_{2 N}^{(l)}(\omega)$ is now an $[(N-1) / N]$-Padé approximant ${ }^{17}$ to Eq. (A5) that reproduces the low-frequency moments $\mu_{-1}$ to $\mu_{-2 \mathrm{~N}}$. Hence, it is an equivalent functional form of the approximant $\bar{c}_{N}(\omega)$ of Sec. II.

\section{APPENDIX B: EQUIVALENT REDUCED HAMILTONIAN}

The starting point of our considerations here is the demonstration that our approximant $\bar{c}_{N}(\omega)$ can also be written in a functional form which had previously been found for a high-frequency expansion':

$$
\bar{c}_{N}(\omega)=\mathbf{a}_{N}^{+}\left[\omega \mathbf{A}_{0}+\mathbf{A}_{1}\right]^{-1} \mathbf{a}_{N},
$$

where $\mathrm{A}_{0}$ and $\mathrm{A}_{1}$ are $N \times N$ matrices, $\mathrm{a}_{N}$ is an $N$ vector and $\mathbf{a}_{N}^{+}$is its transpose. In our case, which is a low-frequency expansion case, the elements of these quantities are given by $(i, j=0, \ldots, N-1)$

$$
\begin{aligned}
& \left(\mathbf{a}_{n}\right)_{j}=\mu_{-2 N+n+j} \quad \text { with } n=0, \ldots, N, \\
& \left(\mathbf{A}_{0}\right)_{i j}=\mu_{-2 N+i+j}, \quad \text { i.e., } \mathbf{A}_{0}=\left(\mathbf{a}_{0}, \mathbf{a}_{1}, \ldots, \mathbf{a}_{N-1}\right), \\
& \left(\mathbf{A}_{1}\right)_{i j}=\mu_{-2 N+i+j+1}, \quad \text { i.e., } \mathbf{A}_{1}=\left(\mathbf{a}_{1}, \mathbf{a}_{2}, \ldots, \mathbf{a}_{N}\right) .
\end{aligned}
$$

We first establish Eq. (B1) by showing it reproduces the low-frequency moments $\mu_{-n}, n=1, \ldots, 2 N$, of a function with the asymptotic expansion (2.11). ${ }^{18}$

\section{Equivalence of low-frequency moments}

A straightforward calculation shows that the formal expansion of Eq. (B1) in powers of $\omega$ is

$$
\bar{c}_{N}(\omega) \sim \sum_{n=0}(-\omega)^{n}\left[\mathbf{a}_{N}^{+} \mathbf{A}_{1}^{-1}\left(\mathbf{A}_{0} \mathbf{A}_{1}^{-1}\right)^{n} \mathbf{a}_{N}\right] .
$$

Hence, by comparison with Eq. (2.11), the low-frequency moments $\mu_{-n}^{\prime}$ in Eq. (B3) are given by

$$
\mu_{-n}^{\prime}=\mathbf{a}_{N}^{+} \mathbf{A}_{1}^{-1}\left(\mathbf{A}_{0} \mathbf{A}_{1}^{-1}\right)^{n-1} \mathbf{a}_{N} .
$$

From the structure of $\mathbf{A}_{1}$, Eq. (B2c), one can deduce that $\mathbf{a}_{v}$ equals $A_{1} e_{v-1}$, with $e_{v}$ being the unity vector in direction $v$,

$$
\left(\mathbf{e}_{v}\right)_{j}=\delta_{j v} .
$$

Hence,

$$
\mathbf{A}_{1}^{-1} \mathbf{a}_{v}=\mathbf{e}_{v-1} \text { for } 0<v \leqslant N .
$$

Employing these results one sees immediately that

$$
\left(\mathbf{A}_{0} \mathbf{A}_{1}^{-1}\right)^{v} \mathbf{a}_{N}=\mathbf{a}_{N-v} \quad \text { for } 0 \leqslant v \leqslant N
$$

holds. Since $A_{0}$ and $A_{1}$ are symmetric, Eqs. (B6) and (B7) hold also in their transposed form, i.e.,

$$
\mathbf{a}_{v}^{+} \mathbf{A}_{1}^{-1}=\mathbf{e}_{v-1}^{+} \quad \text { for } 0<v \leqslant N
$$

and

$$
\mathbf{a}_{N}^{+}\left(\mathbf{A}_{1}^{-1} \mathbf{A}_{0}\right)^{\nu}=\mathbf{a}_{N-v}^{+} \text {for } 0 \leqslant v \leqslant N .
$$

Applying these relations one can deduce that

$$
\mathbf{a}_{N}^{+} \mathbf{A}_{1}^{-1}\left(\mathbf{A}_{0} \mathbf{A}_{1}^{-1}\right)^{v}=\mathbf{e}_{N-(v+1)}^{+} \text {for } 0 \leqslant v<N .
$$

Since every integer $n$ with $0<n \leqslant 2 N$ can be split into integers $v_{1}$ and $v_{2}$ with $n=v_{1}+v_{2}+1, v_{1}$ lying in the interval $[0, N-1]$ and $v_{2}$ lying in the interval $[0, N]$, we have 


$$
\begin{aligned}
\mu_{-n}^{\prime} & =\mathbf{a}_{N}^{+} \mathbf{A}_{1}^{-1}\left(\mathbf{A}_{0} \mathbf{A}_{1}^{-1}\right)^{v_{1}}\left(\mathbf{A}_{0} \mathbf{A}_{1}^{-1}\right)^{v_{2}} \mathbf{a}_{N} \\
& =\mathbf{e}_{N-\left(v_{1}+1\right) \cdot \mathbf{a}_{N-v_{2}}} \\
& =\mu_{-n} \quad \text { for } n=1, \ldots, 2 N,
\end{aligned}
$$

which completes the proof.

\section{Positive definiteness of the matrix $A_{0}$}

We next show that the matrix $\mathbf{A}_{0}$ is positive definite. This property can be established by utilizing an equivalent representation of the moments through a spectral expansion of $\mathbf{H}$, using Eqs. (2.3) and (2.12),

$$
\mu_{-n}=\sum_{k} b_{k}\left(E_{k}-\langle\mathbf{H}\rangle\right)^{-n}
$$

Using Eq. (B10) we obtain for an arbitrary vector $\mathbf{x}$,

$$
\begin{aligned}
\mathbf{x}^{+} \mathbf{A}_{0} \mathbf{x}= & \sum_{i, j=0}^{N-1} x_{i} x_{j} \mu-2 N+i+j \\
= & \sum_{k} b_{k}\left(E_{k}-\langle\mathbf{H}\rangle\right)^{-2 N} \sum_{i, j=0}^{N-1} x_{i} x_{j}\left(E_{k}-\langle\mathbf{H}\rangle\right)^{i+j} \\
= & \sum_{k} b_{k}\left(E_{k}-\langle\mathbf{H}\rangle\right)^{-2 N} \\
& \times\left[\sum_{i=0}^{N-1} x_{i}\left(E_{k}-\langle\mathbf{H}\rangle\right)^{i}\right]^{2}>0 .
\end{aligned}
$$

The inequality in the last line holds since all factors in the sum are positive [e.g., $b_{j}$ is given by Eq. (2.3b)], and not all terms in the sum vanish simultaneously. ${ }^{19}$ We note that such a property does not hold for the matrix $\mathbf{A}_{1}$ : analogously to Eq. (B11) one obtains

$$
\begin{aligned}
\mathbf{x}^{+} \mathbf{A}_{1} \mathbf{x}= & \sum_{k} b_{k}\left(E_{k}-\langle\mathbf{H}\rangle\right)^{-(2 N+1)} \\
& \times\left[\sum_{i=0}^{N-1} x_{i}\left(E_{k}-\langle\mathbf{H}\rangle\right)^{i}\right]^{2},
\end{aligned}
$$

and, since the term with the odd exponent can be negative, the matrix $\mathbf{A}_{1}$ is not positive definite, in general.

\section{Equivalent reduced Hamiltonian}

Since $\mathbf{A}_{0}$ is symmetric and positive definite there exists a unique symmetric and positive definite matrix $\mathbf{A}_{0}^{1 / 2}$ which is a square root of $\mathbf{A}_{0}$. This property leads to the following symmetric form for $\mathrm{Eq}$. (B1):

$$
\bar{c}_{N}(\omega)=\left(\mathbf{A}_{0}^{-1 / 2} \mathbf{a}_{N}\right)^{+}\left[\omega+\mathbf{H}_{\mathrm{red}}\right]^{-1}\left(\mathbf{A}_{0}^{-1 / 2} \mathbf{a}_{N}\right)
$$

with

$$
H_{\text {red }}=A_{0}^{-1 / 2} A_{1} A_{0}^{-1 / 2} \text {. }
$$

Because of the properties of $A_{0}$ and $A_{1}$ the matrix $H_{\text {red }}$ is real and symmetric and has, therefore, real eigenvalues. Using a spectral expansion of $\mathbf{H}_{\text {red }}$ we regain the form Eq. (2.13a) of $\bar{c}_{N}(\omega)$, i.e.,

$$
\bar{c}_{N}(\omega)=\sum_{j=1}^{N} \frac{c_{j}}{\omega+\epsilon_{j}},
$$

with

$$
c_{j}=\frac{\left(\mathbf{h}_{j}^{+} \mathbf{A}_{0}^{-1 / 2} \mathbf{a}_{N}\right)^{2}}{\left|\mathbf{h}_{j}\right|^{2}},
$$

and the $\epsilon_{j}$ and $\mathbf{h}_{j}$ being the eigenvalues and eigenvectors of $\mathbf{H}_{\text {red }}$. Equation (B15) shows that the parameters $c_{j}$ are real and positive. Hence, the approximation presented in Sec. II always leads to a regular form and no spurious divergencies or negative probabilities can occur.

The above results provide the possibility of calculating the parameters $\epsilon_{j}$ and $c_{j}$ of the present approximation numerically by determining the eigenvalues and eigenvectors of $\mathbf{H}_{\text {red }}$. However, it turns out that this process is still somewhat complicated since one has to determine first the square root matrix $\mathbf{A}_{0}^{1 / 2}$ and its inverse. By a further transformation of Eq. (B1) we can arrive at a still simpler eigenvalue problem presented in Appendix $\mathrm{C}$, which is the method used in this paper to determine the $c_{j}$ 's and $\epsilon_{j}$ 's.

Equation (B13) leads also to an interpretation of our approximation in terms of an equivalent reduced Hamiltonian given by the matrix $\mathbf{H}_{\text {red }}$, Eq. (B14). $\mathbf{H}_{\text {red }}$ governs the evolution of an equivalent "reduced" nonstationary state $\left|\psi_{\text {red }}(t)\right\rangle$ that is coupled to a system of $(N-1)$ virtual states which describe the influence of the rest of the quantum system. $c_{N}(t)$ is the probability amplitude for $\left|\psi_{\text {red }}(t)\right\rangle$ to have still its initial value $\left|\psi_{\text {red }}(0)\right\rangle$, i.e.,

$c_{N}(t)=\left\langle\psi_{\text {red }}(0) \mid \psi_{\text {red }}(t)\right\rangle=\left\langle\psi_{\text {red }}(0)\left|e^{-i \mathbf{H}_{\text {red }} t}\right| \psi_{\text {red }}(0)\right\rangle$,

The virtual states and their interactions in $\mathbf{H}_{\text {red }}$ are tailored in such a way that the low-frequency properties of the exact $C(t)$ are reproduced.

\section{Properties of the reduced initial state}

The reduced initial state $\left|\psi_{\text {red }}(0)\right\rangle$ is represented in the reduced Hamiltonian description (B13) by the vector

$$
\left|\psi_{\text {red }}(0)\right\rangle=\mathbf{A}_{0}^{-1 / 2} \mathbf{a}_{N}
$$

We note that this state is not necessarily normalized to unity, i.e.,

$$
\left|\psi_{\text {red }}(0)\right|^{2}=\mathbf{a}_{N}^{+} \mathbf{A}_{0}^{-1} \mathbf{a}_{N} \leqslant 1 \text {. }
$$

A proof of the inequality is given in Appendix D. Equation (B18) is related to the fact that in the original problem, averaged over long times, probability is lost into the remaining states of the quantum system. The present low-frequency approximation, represented by $c_{N}(t)$ in Eq. (2.13b), takes this property already into account by giving the effective initial state a probability that can be less than unity. In the effective Hamiltonian approach of Ref. 8, this property has been taken into account by use of additional phenomenological correction factors. ${ }^{8 b}$

For $N=1$, the initial value, given by Eq. (B18), is already an estimate for the time-average $\bar{p}$, Eq. (2.17), of the occupation probability of this state. It can be shown to assume a particularly simple form. To do so we write the Hamiltonian $\delta \mathbf{H}$ in the form

$$
\delta \mathbf{H}=\left(\begin{array}{cc}
0 & \mathbf{v}^{+} \\
\mathbf{v} & \delta \mathbf{H}_{B}
\end{array}\right)
$$

with $\nabla$ denoting the interaction of the nonstationary state with the other states of the quantum system (which can be viewed as bath states). $\delta \mathbf{H}_{B}$ is the Hamiltonian of this bath. In the notation of Eq. (B19) the initial nonstationary state is 
the vector given by $|\psi(0)\rangle=\left(\begin{array}{l}1 \\ 0\end{array}\right)$. After determining the moments in terms of $\delta \mathbf{H}_{B}$ we obtain for the initial probability

$$
\left|\psi_{\text {red }}(0)\right|^{2}=\frac{1}{1+\mathbf{v}^{+} \delta \mathbf{H}_{B}^{-2} \mathbf{v}} .
$$

This result can be related to the phenomenological correction factor $f_{R}$ derived in Ref. 8(b). For the partitioning (B19) of the Hamiltonian this correction factor assumes the form

$$
f_{R}=1-\mathbf{v}^{+} \delta \mathbf{H}_{B}^{-2} \mathbf{v},
$$

which follows also from Eq. (B20) upon expanding the denominator and neglecting higher-order terms.

The examples presented in Sec. III show that the $N=1$ approximation (B20) to the initial probability gives good results for the time-average $\bar{p}$ of the occupation probability, Eq. (2.17), in cases where no states resonant with the initial state are present. It fails, however, when resonant states are present. Nevertheless, already the second-order approximation determines $\bar{p}$ reasonably well so that the long-time average of the occupation probability is reproduced correctly together with the amplitude of the low-frequency quantum beats. We note that the generalization of Eq. (B21) for resonant subspaces derived in Ref. 8(b) cannot be compared to the present higher-order approximations, since $f_{R}$ determines the total probability of the full resonant subspace, whereas in our case only the reduced probability of the initial state is considered.

\section{APPENDIX C: EQUIVALENT EIGENVALUE PROBLEM}

\section{We define the matrix}

$$
\mathbf{F}=\mathbf{A}_{0}^{-1 / 2} \mathbf{H}_{\text {red }} \mathbf{A}_{0}^{1 / 2}=A_{0}^{-1} \mathbf{A}_{1} \text {. }
$$

With this matrix, Eqs. (B1) and (B13) assume the form

$$
\bar{c}_{N}(\omega)=\mathbf{a}_{N}^{+}[\omega+\mathbf{F}]^{-1} \mathbf{A}_{0}^{-1} \mathbf{a}_{N} .
$$

From the structure of $\mathbf{A}_{0}$ we can deduce, similarly as for $\mathrm{Eq}$. (B6), that

$$
\mathbf{A}_{0}^{-1} \mathbf{a}_{v}=\mathbf{e}_{v+1} \quad \text { for } 0 \leqslant v<N
$$

holds, and we arrive at the result that $\mathrm{F}$ has Frobenius form ${ }^{20}$

$$
\begin{aligned}
\mathbf{F} & =\left(\mathbf{e}_{1}, \ldots, \mathbf{e}_{N-1}, \boldsymbol{\gamma}\right) \\
& =\left(\begin{array}{ccccc}
0 & 0 & \cdots & 0 & \gamma_{0} \\
1 & 0 & \cdots & 0 & \gamma_{1} \\
0 & 1 & \cdots & 0 & \gamma_{2} \\
\vdots & \vdots & \ddots & \vdots & \vdots \\
0 & 0 & \cdots & 1 & \gamma_{N-1}
\end{array}\right),
\end{aligned}
$$

a special form of an upper Hessenberg matrix. ${ }^{13}$ The vector $\gamma$ in Eq. (C4) is the solution of the linear equation

$$
A_{0} \gamma=a_{N} .
$$

Due to its much simpler form the matrix $F$ can be determined more easily than the reduced Hamiltonian $\mathbf{H}_{\mathrm{red}}$. Because of the relation ( $\mathrm{Cl}$ ) between $\mathrm{F}$ and $\mathrm{H}_{\mathrm{red}}$, both matrices have the same eigenvalues $\epsilon_{j}$ and their (right) eigenvectors are related by

$$
\mathbf{h}_{j}=\mathbf{A}_{0}^{1 / 2} \mathbf{f}_{j} \text {. }
$$

Due to the specific form of $\mathbf{F}$, its right eigenvectors $\mathbf{f}_{j}$ can be calculated in a particularly simple iterative way once the eigenvalues $\epsilon_{j}$ are known,

$$
\begin{aligned}
& \left(\mathbf{f}_{j}\right)_{0}=\gamma_{0} / \epsilon_{j}, \\
& \left(\mathbf{f}_{j}\right)_{i}=\left[\left(\mathbf{f}_{j}\right)_{i-1}+\gamma_{i}\right] / \epsilon_{j} \text { for } i=1, \ldots, N-1 .
\end{aligned}
$$

Replacing the vectors $h_{j}$ in Eq. (B15) through $\mathbf{f}_{j}$ via Eq. (C6) we obtain

$$
\bar{c}_{N}(\omega)=\sum_{j=1}^{N} \frac{c_{j}}{\omega+\epsilon_{j}},
$$

with

$$
c_{j}=\frac{\left(\mathbf{a}_{N} \cdot \mathbf{f}_{j}\right)^{2}}{\left(\mathbf{f}_{j}^{+} \mathbf{A}_{0} \mathbf{f}_{j}\right)} .
$$

Hence, for the actual determination of the parameters $\epsilon_{j}$ and $c_{j}$ the simplest way is to determine the matrix $\mathbf{F}$ first and calculate its eigenvalues numerically. From this result the determination of the eigenvectors $\mathbf{f}_{j}$ via Eq. (C7) and of the parameters $c_{j}$ via $\mathrm{Eq}$. (C8) is straightforward.

This procedure is, in fact, the one used in the present paper to determine the approximation in our applications for all $N$, although Eqs. (2.15) and (2.16) could have been used also for $N \leqslant 2$. The matrix $\mathbf{F}$ was determined by numerically solving $\mathrm{Eq}$. (C5) and then diagonalized numerically to determine the $\epsilon_{j}$. The parameters $c_{j}$ were determined from the $\epsilon_{j}$ by Eqs. (C7) and (C8).

\section{APPENDIX D: INNER PROJECTIONS}

A projection operator $\mathbf{Q}_{N}$ that projects onto the $N$-dimensional space spanned by the linearly independent set of states $|\mathbf{q}\rangle=\left(\left|q_{1}\right\rangle, \ldots,\left|q_{N}\right\rangle\right)$ has the form

$$
\mathbf{Q}_{N}=|\mathbf{q}\rangle[\langle\mathbf{q} \mid \mathbf{q}\rangle]^{-1}\langle\mathbf{q}|
$$

with $\langle\mathbf{q} \mid \mathbf{q}\rangle$ denoting the metric matrix of this basis set. An inner projection $\mathbf{R}_{i}$ of a positive definite Hermitian operator $\mathbf{R}$ onto this space is defined by ${ }^{21}$

$$
\mathbf{R}_{i}=\mathbf{R}^{1 / 2} \mathbf{Q}_{N} \mathbf{R}^{1 / 2} \text {. }
$$

Employing the substitution $|\mathbf{q}\rangle=\mathbf{R}^{-1 / 2}|\mathbf{k}\rangle$, the inner projection $\mathbf{R}_{i}$ of the operator $\mathbf{R}$ assumes the form

$$
\mathbf{R}_{i}=|\mathbf{k}\rangle\left[\left\langle\mathbf{k}\left|\mathbf{R}^{-1}\right| \mathbf{k}\right\rangle\right]^{-1}\langle\mathbf{k}|,
$$

known also as Bazley projection. ${ }^{22}$ Inner projections of positive definite operators provide lower bounds to these operators, i.e., for arbitrary states $|x\rangle$ the relation

$$
\left\langle x\left|\mathbf{R}_{i}\right| x\right\rangle \leqslant\langle x|\mathbf{R}| x\rangle
$$

holds. ${ }^{21}$ In particular, $\mathbf{R}_{i}$ as given by Eq. (D3) is an optimal lower bound to $\mathbf{R}$ in the subspace spanned by $|\mathbf{k}\rangle$.

Let now $\mathbf{R}$ be the resolvent operator in Sec. II,

$$
\mathbf{R}=[\omega+\delta \mathbf{H}]^{-1} \text {. }
$$

For $\omega>\omega_{\min }$ with $\omega_{\min }=\left|\min \left(0, E_{0}-\langle\mathbf{H}\rangle\right)\right|, E_{0}$ being the ground-state energy of $\mathbf{H}$, this resolvent operator is positive definite and Hermitian. Furthermore, we choose the linearly independent set of states to be $|\mathbf{k}\rangle$ $=\left[\delta \mathbf{H}^{-N}|\psi(0)\rangle, \ldots, \delta \mathbf{H}^{-1}|\psi(0)\rangle\right]$. Then we obtain (see also Ref. 9) 


$$
\begin{aligned}
\left\langle\psi(0)\left|\mathbf{R}_{i}\right| \psi(0)\right\rangle= & \langle\psi(0) \mid \mathbf{k}\rangle \\
& \times[\langle\mathbf{k}|\omega+\delta \mathbf{H}| \mathbf{k}\rangle]^{-1}\langle\mathbf{k} \mid \psi(0)\rangle \\
= & \mathbf{a}_{N}^{+}\left[\omega \mathbf{A}_{0}+\mathbf{A}_{1}\right]^{-1} \mathbf{a}_{N},
\end{aligned}
$$

i.e., the inner projection of the resolvent operator onto the such chosen subspace leads to the present low-frequency approximation for the Laplace transformed probability amplitude. From Eq. (D4) we see that the inequality

$$
\mathbf{a}_{N}^{+}\left[\omega \mathbf{A}_{0}+\mathbf{A}_{1}\right]^{-1} \mathbf{a}_{N} \leqslant\left\langle\psi(0)\left|[\omega+\delta \mathbf{H}]^{-1}\right| \psi(0)\right\rangle
$$

holds in the domain where the resolvent operator is positive definite, i.e., for $\omega>\omega_{\min }$. In particular, upon multiplying both sides with $\omega$ and taking the limit $\omega \rightarrow \infty$ we derive the inequality in Eq. (B18).

In Ref. 23 it was demonstrated that the inner projection $\mathbf{R}_{i}$ converges to $\mathbf{R}$ for $N \rightarrow \infty$ if the space spanned by $|\mathbf{q}\rangle$ becomes complete. In our case, only the subspace of $\delta \mathbf{H}$ wherein $|\psi(0)\rangle$ evolves is of interest. It is anticipated that $|q\rangle$ should become complete in this subspace when the operator $\delta \mathbf{H}$ is given by an irreducible matrix Hamiltonian. The convergence property holds also for operators $\mathbf{R}$ not positive definite, i.e., for $\omega<\omega_{\min }$ in our case, as long as $\mathbf{R}$ is nonsingular. ${ }^{23} \mathbf{R}$ as given by Eq. (D5) is singular only for a finite number of values $\omega$, corresponding to the negative eigenvalues of $\delta \mathbf{H}$. Hence, the convergence of our approximation to the exact Laplace-transformed probability amplitude is guaranteed for almost all values of $\omega$. Although this convergence can be only a pointwise convergence and not a uniform convergence (which would have given the possibility of deriving error bounds), it nevertheless assures that the present method is also reliable with respect to ultimate convergence to the exact solution.

'W. R. Lambert, P. M. Felker, and A. H. Zewail, J. Chem. Phys. 75, 5958 (1981); P. M. Felker and A. H. Zewail, Phys. Rev. Lett. 53, 501 (1984). ${ }^{2}$ P. M. Felker and A. H. Zewail, J. Chem. Phys. 82, 2961, 2975 (1985).

${ }^{3}$ We give here a long, but by no means exhaustive, list of works dealing with this problem, see also Refs. 6-8 below. K. F. Freed, Top. Appl. Phys. 15, 23 (1976); P. Avouris, W. M. Gelbart, and M. A. El-Sayed, Chem. Rev. 77, 793 (1977); S. Mukamel and J. Jortner, in Excited States, edited by E. C. Lim (Academic, New York, 1977) Vol. 3, p. 57; W. Rhodes, J. Phys. Chem. 87, 30 (1983); V. E. Bondybey, Annu. Rev. Phys. Chem. 35, 591 (1984); F. F. Crim, ibid. 35, 657 (1984); E. B. Stechel and E. J. Heller, ibid. 35, 563 (1984); R. E. Smalley, J. Phys. Chem. 86, 3504 (1982); M. L.
Sage and J. Jortner, Adv. Chem. Phys. 47, 293 (1981); S. A. Rice, ibid. 47, 117 (1981); P. R. Stannard and W. M. Gelbart, J. Phys. Chem. 85, 3592 (1981); H. R. Dübal and M. Quack, Chem. Phys. Lett. 80, 439 (1981); E. L. Sibert, W. P. Reinhardt, and J. T. Hynes, J. Chem. Phys. 81, 1115 (1984); J. S. Hutchinson, J. T. Hynes, and W. P. Reinhardt, Chem. Phys. Lett. 108, 353 (1984); J. S. Hutchinson, E. L. Sibert, and J. T. Hynes, J. Chem. Phys. 81, 1314 (1984).

${ }^{4}$ A. Zewail, Faraday Discuss. Chem. Soc. 75, 315 (1983).

${ }^{5}$ J. Cullum and R. A. Willoughby, Lanczos Algorithm for Large Symmetric Eigenvalue Computations I, II (Birkhäuser, Boston, 1985).

${ }^{6}$ A. Nauts and R. E. Wyatt, Phys. Rev. Lett. 51, 2238 (1983); Phys. Rev. A 30, 872 (1984); I. Schek and R. E. Wyatt, J. Chem. Phys. 83, 3028, 4650 (1985); 84, 4497 (1986); K. Milfeld, J. Castillo, and R. E. Wyatt, ibid. 83, 1617,4650 (1985).

7J. V. Tietz and S.-I. Chu, Chem. Phys. Lett. 101, 446 (1983); J. Chang and R. E. Wyatt, ibid. 121, 307 (1985); J. Chem. Phys. 85, 1826 (1986).

${ }^{8}$ (a) G. A. Voth and R. A. Marcus, J. Chem. Phys. 84, 2254 (1986); (b) S. J. Klippenstein, G. A. Voth, and R. A. Marcus, ibid. 85, 5019 (1986); (c) G. A. Voth, Chem. Phys. Lett. 129, 315 (1986).

${ }^{9} \mathrm{O}$. Goscinski and E. Brändas, Int. J. Quantum Chem. 5, 131 (1971).

${ }^{10}$ (a) K. Schulten, A. Brünger, W. Nadler, and Z. Schulten, in Synergetics-From Microscopic to Macroscopic Order, edited by E. Frehland (Springer, Berlin, 1984), pp. 80-89; (b) W. Nadler and K. Schulten, J. Chem. Phys, 82, 151 (1985); (c) 84, 4015 (1986).

"(a) W. Nadler and K. Schulten, Phys. Rev. Lett. 51, 1712 (1983); (b) Z. Phys. B 59, 53 (1985); (c) A. Brünger, R. Peters, and K. Schulten, J. Chem. Phys. 82, 2147 (1985).

${ }^{12}$ H. Risken, The Fokker-Planck Equation (Springer, Berlin, 1984).

${ }^{13}$ W. H. Press, B. P. Flannery, S. A. Teukolsky, and W. T. Vetterling, $\mathrm{Nu}$ merical Recipes (Cambridge University, Cambridge, 1986), and references cited therein.

${ }^{14}$ R. G. Gordon, J. Math. Phys. 9, 65 (1968).

${ }^{15}$ We note, in passing, that an odd approximant $C_{2 N+1}^{(h)}(\omega)$ constitutes an $[N / N]$-Padé approximant.

${ }^{16} \mathrm{R}$. Zwanzig, in Boulder Lectures in Theoretical Physics III, edited by W. E. Brittin, B. W. Downs, and J. Downs (Wiley-Interscience, New York, 1961 ), p. 106; H. Mori, Prog. Theor. Phys. 34, 399 (1965).

${ }^{17}$ As was the case for $C^{(h)}(\omega)$, an odd approximant $C_{2 N+1}^{(l)}(\omega)$ represents an $[N / N]$-Padé approximant. However, these are not of interest in our case since they would lead to a finite nonzero value of the approximant for $\omega \rightarrow \infty$, corresponding to an irregular, $\delta$-function like, short-time behavior.

${ }^{18}$ We strongly follow here in Appendix B 1 and B 2, and in the following Appendix C, the proof and derivation in Ref. 11(c). We note that most of the results stated there are valid also in our case. The proof presented there, however, has to be modified to apply in our case.

${ }^{19}$ The term in square brackets in the last line of Eq. (B11) is an $(N-1)$ th-order polynomial in $\left(E_{k}-\langle\mathbf{H}\rangle\right), x_{0}+x_{1}\left(E_{k}-\langle\mathbf{H}\rangle\right)$ $+\cdots+x_{N-1}\left(E_{k}-\langle\mathrm{H}\rangle\right)^{N-1}$, which has at most $N-1$ different zeros. Hence, the sum over all $k$ terms in Eq. (B11) has at most $N-1$ terms equal to zero. As long as $N-1$ is less than the number of eigenstates there are still nonvanishing positive terms leading to the inequality in Eq. (B11).

${ }^{20}$ The eigenvalues of the Frobenius matrix $(\mathrm{C} 4)$ are the roots of the related polynomial in $\epsilon, \epsilon^{N}-\gamma_{N-1} \epsilon^{N-1}-\cdots-\gamma_{1} \epsilon-\gamma_{0}$.

${ }^{21} P$. O. Löwdin, Int. J. Quantum Chem. 4, 231 (1971).

${ }^{22}$ N. W. Bazley, Phys. Rev. 120, 144 (1960).

${ }^{23}$ P. O. Löwdin, Int. J. Quantum Chem. 21, 69 (1982). 